\title{
Introduction to the Minitrack on Truth and Lies: Deception and Cognition on the Internet
}

\author{
Randall K. Minas \\ University of Hawai'i at Manoa \\ rminas@hawaii.edu
}

\author{
Alan R. Dennis \\ Indiana University \\ ardennis@,indiana.edu
}

Recent events demonstrate a need for greater research on deception, biases, and trust on the Internet. Over the past year, the topic of fake news and its consequences have become omnipresent in the media and on social networking sites, but this is not a new issue. Fake reviews of products and services, fake social media accounts and posts, and fake advertising have long been a feature of the Internet. The effects of deception on businesses and society are tangible in terms of decreased brand image, sales, and loss of trust.

This minitrack examines deception on the Internet, and why individuals fall prey to it. Information selection biases, such as confirmation bias, affect the types of information that individuals process. These selection biases inform how individuals structure their attitudes and create their perceptions of reality. This minitrack focuses on information that is demonstrably false but provided on the Internet in order to manipulate an individual's attitudes towards a business, product, or other societal issues. It also takes the lens of the individual's response to the deception from bias in processing the information to the actions individuals take after they have received the information.

Several high-quality submissions were received for the inaugural minitrack. We hope to offer this minitrack for in HICSS to come as the continued evolution of deception on the Internet calls for increased research on the topic area. For the inaugural minitrack, we have four papers organized into one session.

Says Who?: How News Presentation Format Influences Perceived Believability and the Engagement
Level of Social Media Users by Antino Kim and Alan Dennis investigates the format of news presentation at its effect on perceived believability and engagement level in social media. The findings provide insights into how the presentation format affects how the users perceive and, subsequently, act on news.

Disruption and Deception in Crowdsourcing: Towards a Crowdsourcing Risk Framework by Agnieszka Onuchowska and Gert-Jan de Vreede examines the emergence of disruptive and deceptive issues in crowdsourcing. The authors create a framework for the efforts to deceive in the crowdsourcing arena.

Combating Fake News: An Investigation of Information Verification Behaviors on Social Networking Sites by Russell Torres, Natalie Gerhart, and Arash Negahban examines how users of social networking sites determine when to check on validity of news. The authors provide several insightful findings on the key drivers for users' news verification behavior.

A Tale of Two Internet News Platforms-Real vs. Fake: An Elaboration Likelihood Model Perspective by Babajide Osatuyi and Jerald Hughes provides a field analysis of real and fake news that is propagated on the Internet using an Elaboration Likelihood Model (ELM) perspective. The authors examine the amount of information disseminated by fake news platforms and reputable news platforms, analyzing the business impact of fake news.

We are pleased to have had such great submissions to the minitrack and look forward to a great discussion on deception and the Internet at HICSS-51! 